\title{
Is the Fair Sampling Assumption supported by EPR Experiments?
}

\author{
Guillaume Adenier, Andrei Yu. Khrennikov \\ International Center for Mathematical Modelling in Physics and Cognitive Sciences \\ Växjö University, 35195 Växjö, Sweden. \\ E-mail: guillaume.adenier@vxu.se
}

\begin{abstract}
We analyze optical EPR experimental data performed by Weihs et al. in Innsbruck 1997-1998. We show that for some linear combinations of the raw coincidence rates, the experimental results display some anomalous behavior that a more general source state (like non-maximally entangled state) cannot straightforwardly account for. We attempt to explain these anomalies by taking account of the relative efficiencies of the four channels. For this purpose, we use the fair sampling assumption, and assume explicitly that the detection efficiencies for the pairs of entangled photons can be written as a product of the two corresponding detection efficiencies for the single photons. We show that this explicit use of fair sampling cannot be maintained to be a reasonable assumption as it leads to an apparent violation of the no-signalling principle.
\end{abstract}

PACS numbers: 03.65.-w, 03.65.Ud 


\section{Introduction}

The experimental violation of Bell Inequalities $[1,2,3,4]$ in optical EPR experiments $[5,6]$ can only be validated under the additional assumption of fair sampling $[4,7,8]$. This type of test being crucial for modern quantum communication research $[9,10]$, it is unfortunate that the result of a test meant to disprove Local Realism would depend on such an additional assumption, since Local Realism is a priori no less plausible an assumption than Fair Sampling is.

In this article we examine data from past experiments and look for possible traces of failure of the Fair Sampling assumption. For this purpose, we were recently given the chance to examine raw data from the long distance experiment with fast switching performed in Innsbruck in 1997-1998 by Gregor Weihs et al. We show that the near perfect agreement with the predictions of Quantum Mechanics obtained from experiments can be questioned when extracted data are looked from a different perspective, that is, when comparing other linear combinations of the normalized coincidence rates than the correlation function with the predictions of Quantum Mechanics.

\section{Channel efficiencies}

In a real experiment, not all photons are detected and one should take account of the efficiencies of each of the four channels involved. This problem is at the heart of the detection efficiency loophole, and can be used to design local realistic models that reproduce the main experimental features of optical EPR experiments [11].

We label $\eta_{A}^{+}(\alpha)$ and $\eta_{A}^{-}(\alpha)$ the single-count channel efficiencies of Alice's plus and minus channels respectively, and similarly $\eta_{B}^{+}(\beta)$ and $\eta_{B}^{-}(\beta)$ for Bob's plus and minus channels; the parameter dependence in $\alpha$ and $\beta$ reflects the fact that these efficiencies may, in principle, depend on the local settings. It is important to stress that we can put in the concept of channel efficiencies all possible variations in the experimental conditions that are not directly related to the quantum state associated to the source (such as signal intensity variations, detector efficiencies, optical misalignments, collection efficiencies, etc.). This idea is important since it is precisely the aim of our paper to analyze experimental quantities that can directly be compared with Quantum predictions - under the assumption of Fair Sampling — independently of all these experimental contingencies encapsulated in the channel efficiencies.

We can expect the number of single counts to be proportional to the respective channel efficiencies:

$$
\begin{aligned}
& N_{\exp }^{A, \varepsilon_{1}}(\alpha) \approx \eta_{A}^{\varepsilon_{1}}(\alpha) N_{A} / 2 \\
& N_{\exp }^{B, \varepsilon_{2}}(\beta) \approx \eta_{B}^{\varepsilon_{2}}(\beta) N_{B} / 2,
\end{aligned}
$$

where $N_{A}$ and $N_{B}$ are the (unknown) number of photons actually sent respectively to Alice and Bob, and where $\varepsilon_{1}$ and $\varepsilon_{2}$ can each be either + or - , to shorten the forthcoming 
equations $\ddagger$.

We now consider the number of coincidence counts experimentally registered. If we consider a pair of photons as a whole, the probability that it is detected in each specific combination of two channels should depend as well on a combined channel efficiency. We label these combined channel efficiencies as $\eta^{++}(\alpha, \beta), \eta^{+-}(\alpha, \beta), \eta^{-+}(\alpha, \beta)$, and $\eta^{--}(\alpha, \beta)$. The number of coincidences in a pair of channels $\left(\varepsilon_{1}, \varepsilon_{2}\right)$ should thus be proportional to the relevant combined efficiency $\eta^{\varepsilon_{1}, \varepsilon_{2}}(\alpha, \beta)$, the (unknown) number of pairs sent $N_{A B}$, and the relevant joint probability predicted by Quantum Mechanics $P_{Q T}^{\varepsilon_{1}, \varepsilon_{2}}(\alpha, \beta)$, that is,

$$
N_{\exp }^{\varepsilon_{1}, \varepsilon_{2}}(\alpha, \beta) \approx \eta^{\varepsilon_{1}, \varepsilon_{2}}(\alpha, \beta) N_{A B} P_{Q T}^{\varepsilon_{1}, \varepsilon_{2}}(\alpha, \beta) .
$$

Here the angular dependence of the efficiencies is clearly unwanted since we are interested in an experimental test of the predictions of Quantum Mechanics, independently of the inaccuracies involved in any particular experimental setup. Our immediate purpose is therefore to get rid of these angular dependencies due to the combined efficiencies.

In order to do so, we assume that the ensemble of detected pairs of photon provides a fair statistical sample of the ensemble of emitted pairs (Fair Sampling Assumption). A consequence of this assumption is that the probabilities of non detection for Alice and Bob should be independent of one another. Indeed, for fixed settings $(\alpha, \beta)$, the probability of a non detection should be independent of the polarization state of the photon (otherwise the sampling would clearly be unfair), and should thus be independent of the fate of the distant correlated photon.

Hence, the channel efficiencies should be the same for all photons going into a specific channel, independently of whether a photon happens to be single or to be paired with a distant detected photon. That is, the above combined efficiencies for pairs of particles should be equal to the product of the relevant channel efficiencies for the single counts:

$$
\eta^{\varepsilon_{1}, \varepsilon_{2}}(\alpha, \beta)=\eta_{A}^{\varepsilon_{1}}(\alpha) \eta_{B}^{\varepsilon_{2}}(\beta) .
$$

With this factorization of the channel efficiencies, we can rewrite the predicted number of coincidence counts as:

$$
N_{\exp }^{\varepsilon_{1}, \varepsilon_{2}}(\alpha, \beta) \approx N_{A B} \eta_{A}^{\varepsilon_{1}}(\alpha) \eta_{B}^{\varepsilon_{2}}(\beta) P_{Q T}^{\varepsilon_{1}, \varepsilon_{2}}(\alpha, \beta)
$$

\section{Normalizing using the single counts}

The probabilities derived from the standard normalization procedure with coincidence counts still depend explicitly on the channel efficiencies, so that some properties of the photon pairs will remain out of reach of the experimenter.

True enough, in some specific cases the standard normalization procedure can make the channel efficiencies disappear from the final result. For instance, if on one side the

$\ddagger$ The use of the symbol $\approx$ is here to remind of the statistical variability in any experimental results that is naturally expected to induce small deviation from the predictions. The amplitude of these deviations are expected to decrease with the number of trials obtained experimentally. 
channels are balanced, (e.g. $\eta_{B}^{+}(\beta)=\eta_{B}^{-}(\beta)$ ), it is straightforward to show that the standard normalization with the total sum of coincidences removes the channel efficiency dependence of the correlation function:

$$
\frac{N_{\exp }^{++}+N_{\exp }^{--}-N_{\exp }^{+-}-N_{\exp }^{-+}}{\sum_{\varepsilon_{1}, \varepsilon_{2}} N_{\exp }^{\varepsilon_{1}, \varepsilon_{2}}} \approx P_{Q T}^{++}+P_{Q T}^{--}-P_{Q T}^{+-}-P_{Q T}^{-+}=E_{Q T} .
$$

However, for other linear combination of the coincidence counts, the normalization by the total sum of coincidences cannot remove the channel efficiency dependence, even if on one side the channels are balanced. For instance, if $\eta_{B}^{+}(\beta)=\eta_{B}^{-}(\beta)$, then:

$$
\frac{N_{\exp }^{++}+N_{\exp }^{+-}}{\sum_{\varepsilon_{1}, \varepsilon_{2}} N_{\exp }^{\varepsilon_{1}, \varepsilon_{2}}} \approx \frac{\eta_{A}^{+}}{\eta_{A}^{+}+\eta_{A}^{-}}\left(P_{Q T}^{++}+P_{Q T}^{+-}\right) \neq\left(P_{Q T}^{++}+P_{Q T}^{+-}\right) .
$$

This problem makes it necessary to circumvents these dependencies in all cases by means of the new normalization procedure. Our idea is that most of the counts registered in the channels are non-coincidence events, because the channel efficiencies are low. Since there are many times more non-coincident events than coincident ones, they provide a useful and accurate additional statistical information about the relative efficiency of the channels.

In order to get rid of channel efficiencies in the above equations, we thus define the following experimental quantities, which are proportional to the ratio of the number of coincidence counts in a combined channel over the product of the two corresponding single counts:

$$
f_{\exp }^{\varepsilon_{1}, \varepsilon_{2}}(\alpha, \beta) \equiv \frac{1}{4} \frac{N_{\exp }^{\varepsilon_{1}, \varepsilon_{2}}(\alpha, \beta)}{N_{\exp }^{A, \varepsilon_{1}}(\alpha) N_{\exp }^{B, \varepsilon_{2}}(\beta)}
$$

Replacing the single counts and coincidence counts by their expressions given respectively in (1) and (3), we obtain:

$$
f_{\exp }^{\varepsilon_{1}, \varepsilon_{2}}(\alpha, \beta) \approx \frac{N_{A B}}{N_{A} N_{B}} P_{Q T}^{\varepsilon_{1}, \varepsilon_{2}}(\alpha, \beta)
$$

for which the only angular dependence is the one due to the Quantum Mechanical term.

Since the four joint probabilities $P_{Q T}^{++}(\alpha, \beta), P_{Q T}^{+-}(\alpha, \beta), P_{Q T}^{-+}(\alpha, \beta)$ and $P_{Q T}^{--}(\alpha, \beta)$ add up to unity, summing these four equation together yields

$$
\sum_{\varepsilon_{1}, \varepsilon_{2}} f_{\exp }^{\varepsilon_{1}, \varepsilon_{2}}(\alpha, \beta) \approx \frac{N_{A B}}{N_{A} N_{B}}
$$

and finally,

$$
P_{Q T}^{\varepsilon_{1}, \varepsilon_{2}}(\alpha, \beta) \approx \frac{f_{\exp }^{\varepsilon_{1}, \varepsilon_{2}}(\alpha, \beta)}{\sum_{\varepsilon_{1}, \varepsilon_{2}} f_{\exp }^{\varepsilon_{1}, \varepsilon_{2}}(\alpha, \beta)}
$$

Since the normalization procedure that we propose here is different than the standard one based on the coincidence counts alone, a word on the relevance of Eq.(9) with experiments might be necessary here. The validity of Eq.(9) as an approximation of quantum probabilities by experimental frequencies (based on the single counts) depends only on the validity of the Fair Sampling Assumption, an assumption routinely made in 
EPR experiments exhibiting a violation of Bell inequalities§. Indeed, Eq. (2) is but a consequence of this assumption, and leads straightforwardly to Eqs. (6), (9), and (10).

Hence, by using the Fair Sampling Assumption together with experimental statistics for the single counts, we are able to obtain experimental quantities that should coincide with the prediction given by Quantum Mechanics for the joint probabilities, independently of any channel efficiency imbalance. As far as we know, this procedure is new and offers new perspectives for comparing experimental result with Quantum predictions.

In particular this new normalization procedure allows us to obtain experimental quantities that should directly coincide with the marginal probabilities:

$$
\begin{aligned}
& \frac{f_{\exp }^{++}(\alpha, \beta)+f_{\exp }^{+-}(\alpha, \beta)}{\sum_{\varepsilon_{1}, \varepsilon_{2}} f_{\exp }^{\varepsilon_{1}, \varepsilon_{2}}(\alpha, \beta)} \approx P_{Q T}^{++}(\alpha, \beta)+P_{Q T}^{+-}(\alpha, \beta) \\
& \frac{f_{\exp }^{-+}(\alpha, \beta)+f_{\exp }^{--}(\alpha, \beta)}{\sum_{\varepsilon_{1}, \varepsilon_{2}} f_{\exp }^{\varepsilon_{1}, \varepsilon_{2}}(\alpha, \beta)} \approx P_{Q T}^{-+}(\alpha, \beta)+P_{Q T}^{--}(\alpha, \beta) \\
& \frac{f_{\exp }^{++}(\alpha, \beta)+f_{\exp }^{-+}(\alpha, \beta)}{\sum_{\varepsilon_{1}, \varepsilon_{2}} f_{\exp }^{\varepsilon_{1}, \varepsilon_{2}}(\alpha, \beta)} \approx P_{Q T}^{++}(\alpha, \beta)+P_{Q T}^{-+}(\alpha, \beta) \\
& \frac{f_{\exp }^{+-}(\alpha, \beta)+f_{\exp }^{--}(\alpha, \beta)}{\sum_{\varepsilon_{1}, \varepsilon_{2}} f_{\exp }^{\varepsilon_{1}, \varepsilon_{2}}(\alpha, \beta)} \approx P_{Q T}^{+-}(\alpha, \beta)+P_{Q T}^{--}(\alpha, \beta) .
\end{aligned}
$$

An important prediction of Quantum Mechanics for these marginal probabilities is the no-signalling principle. A correlation $P\left(\varepsilon_{1}, \varepsilon_{2} \mid \alpha, \beta\right)$ is non-signaling if and only if its marginal probabilities are independent of the other side input: $\sum_{\varepsilon_{2}} P\left(\varepsilon_{1}, \varepsilon_{2} \mid \alpha, \beta\right)$ is independent of $\beta$ and $\sum_{\varepsilon_{1}} P\left(\varepsilon_{1}, \varepsilon_{2} \mid \alpha, \beta\right)$ is independent of $\alpha$ [14].

This non-signalling property does not depend on the state of the source of photons. Whatever is the source sent to Alice and Bob, they cannot use it to communicate. Alice's marginal probabilities, represented by the two first equations above, cannot depend on Bob's measurement setting $\beta$. Similarly, Bob's marginal probabilities, represented by the two last equations above, cannot depend on Alice's measurement setting $\alpha$.

Hence, if in a series a measurement only one of these parameter varies (say, $\alpha$ ), then only two of these four quantities can vary accordingly, the other two remaining constant. It is precisely this no-signaling prediction conditioned on the validity of the Fair Sampling Assumption that we want to check here.

$\S$ To some extent, one could argue that John Bell used an implicit Fair Sampling Assumption in deriving his theorem, and that although he discarded the possibility that it would not be fulfilled as very unlikely, it remained however a necessary condition that had to always be assumed, but was never fully tested experimentally. In that sense, the Fair Sampling Assumption is as essential to the whole framework of Bell's theorem as are the Locality assumption and the Realism assumption. 

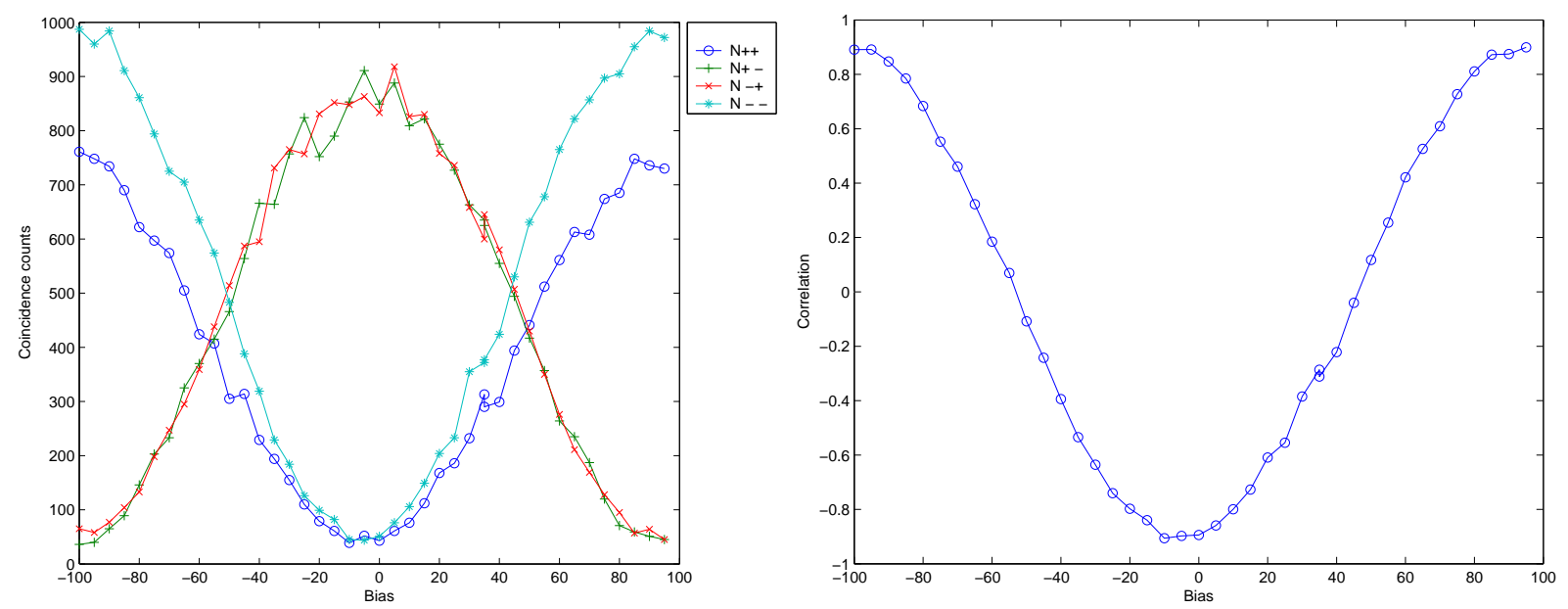

Figure 1. (a) Coincidence counts for a long distance Bell test with fast measurement switching (Innsbruck 1998, source file: scanblue, with switches on position 00),

(b) Correlation Function.

\section{Experimental Results}

\subsection{Long distance with fast switching}

Out of all the various runs we had at hand, we chose first a set of files containing data from a long distance fast switching experiment, with a significant number of coincidences and with enough measurement angles. This run was performed on the 1st of may 1998 in Innsbruck. The files were referred to as Scanblue, meaning that a scan varying "blue" (for Alice) side modulator bias was performed, with both sides randomly fast switching between an equivalent +0 and +45 degrees angle. Modulating the bias from -100 to +100 was linearly equivalent to rotating the corresponding Polarizing Beam splitter from $-\frac{\pi}{2}$ to $+\frac{\pi}{2}$.

We look at the data for the particular case when both switches were set to +0 degrees angle. As can be seen in the coincidence rate figures (see figure 1a), the coincidence rates exhibit minima close to zero, and cosine-squared shape, as expected from the predictions of Quantum Mechanics. However, the maxima of the four coincidence curves differ significantly. In spite of this anomalous behavior, the correlation function computed with the standard normalization (i.e., with the sum of all coincidence counts) coincides very well with the quantum mechanical predictions (see figure $1 b)$.

It is interesting to note that possibly similar anomalies were observed first in the two-channel EPR experiments performed by Alain Aspect in Orsay in the early 1980's. The anomalies were reported in Aspect's PhD Thesis [15] in the following words:

For some measurements, we have observed abnormal differences between coincidence rates that were expected to be equal (for instance $N_{+-}$and $N^{-+}$). It turns out however that even for these measurements the correlation coefficient 


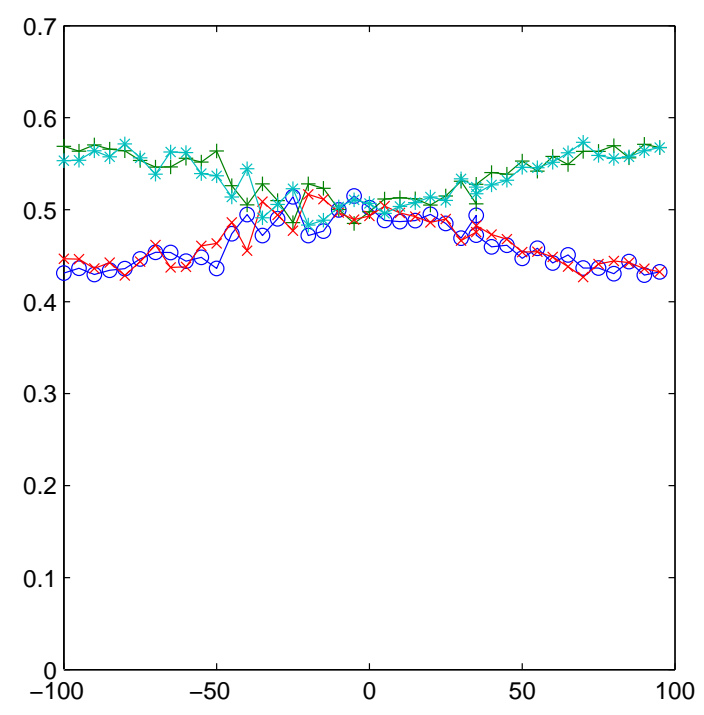

Figure 2. The four possible curves with $\left(N_{\exp }^{\text {even }}+N_{\exp }^{\text {odd }}\right) / \sum_{\varepsilon_{1}, \varepsilon_{2}} N_{\exp }^{\varepsilon_{1}, \varepsilon_{2}}$, showing that the standard normalization with coincidence counts leads to incorrect results.

$E(a, b)$ remains equal to the quantum predictions, with better than two standard deviations. We have no completely convincing explanation, either for these anomalies, or for their compensation $\|$.

This anomalies could in principle be explained by a non-rotationally invariant source state, such as a non-maximally entangled state (see 5). The information available on these anomalies being reduced to the above quotation, it is however impossible to give a definite explanation for them. These anomalies are nevertheless interesting since they indicate that, assuming the state produced by the source was a singlet state and that the channel efficiencies where balanced on each side, the experimental data would not have coincided with the predictions of Quantum Mechanics for all possible linear combinations of the normalized coincidence counts, although the fit with the correlation function was excellent.

To illustrate this point with our particular set of data, we computed the four possible sum of even normalized coincidence counts with odd normalized coincidence counts (see figure 2). In an ideal setup with balanced channels, these four curve should coincide with Alice's and Bob's marginal probabilities (that is, $1 / 2$ for a singlet state). The result displayed in figure 2 shows clearly that other linear combination of the normalized coincidence counts than the correlation function leads to incorrect results. For this purpose we extract first the single counts. The result is displayed in figure 3 .

\| Our translation. The original text goes as follow: Pour quelques mesures on a observé des différences anormales entre taux de coincidences que l'on attendait égaux (par exemple $N_{+-}$et $N_{-+}$). Mais il se trouve que pour ces mesures, le coefficient de corrélation $E(a, b)$ reste égal la prévision quantique, à mieux que deux écarts-types près. Nous n'avons aucune explication complètement convaincante, ni pour ces anomalies, ni pour leur compensation. 


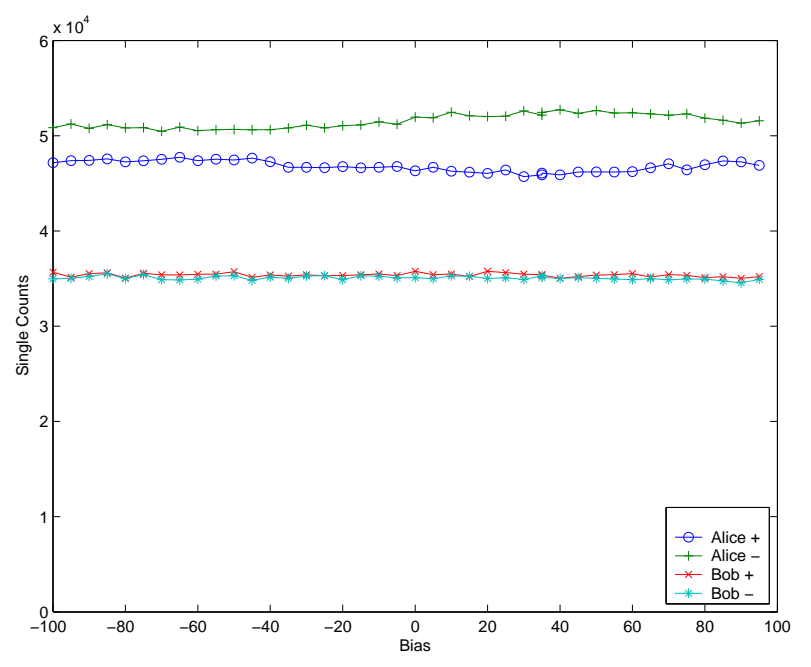

Figure 3. Single Counts (Switches 00, scanblue).

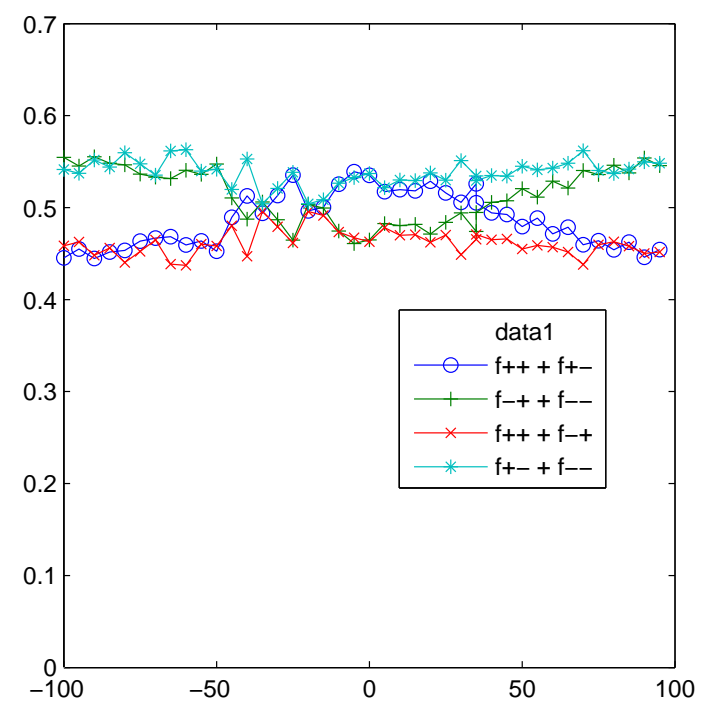

Figure 4. These four quantities $\left(f^{\text {even }}+f^{\text {odd }}\right) / \sum f$ should coincide with Alice's and Bob's marginal probabilities.

As can be seen from the figure, some slight variations of Alice's single count rates can be observed as she varies her bias. It is nevertheless of small amplitude, and remains in any case always local, in the sense that only Alice's single counts vary. More important is the fact that the efficiency of + and - channels differ significantly for Alice.

As can be seen from figure 4, the four experimental quantities expressed by (10) vary with Alice's measurement angle $\alpha$.

For the two first, corresponding to Alice's marginal probabilities, this dependence can be accounted for by a non-rotationally invariant source state, such as a nonmaximally entangled state (see Appendix). However, for the two remaining, there should 
be no angle dependence on $\alpha$ at all, since they should depend only on $\beta$ which remains fixed. The angular dependence of the experimental quantities that should coincide with Bob's marginal probabilities is admittedly weak, but statistical analysis show that a linear fit provides a much poorer fit than a non linear fit for these plots.

Nevertheless, since we had other sets of data available, we decided to investigate whether this anomalous behavior could be exhibited in other experimental setup that would not necessarily be meant to close the locality loophole, but would have cleaner results.

\subsection{Short distance without fast switching}

We thus chose a set of data that have a relatively high maximum number of coincidences and low dark rates, as well as enough different measurement settings, in order to exhibit better the rather tenuous anomaly we observed in all the runs. This run was performed 1997 in Innsbruck. The files were referred to as Bluesine, meaning that only the "blue" side (that is Alice) varied the measurement setting $\alpha$, while the "red" side (that is, Bob) kept the same setting. It had no fast switching and was performed at short distance, but since our goal was not to focus on the locality loophole but rather on detection and sampling issues, this would have been irrelevant anyway.

As can be seen in the coincidence rate figures (see figure 5a), the coincidence rates exhibit minima close to zero, and cosine-squared shape, as expected from the predictions of Quantum Mechanics. However, just like in the long distance experimental setup, the maxima of the four coincidence curves differ significantly.

If we now extract the single-counts, in order to use or normalization procedure, we can see that the efficiency of + and - channels differ significantly for Bob (see figure 5b).

Finally, just like in the long distance setup, the four experimental quantities that should coincide with the marginal probabilities (see figure 6) vary with Alice's measurement angle $\alpha$.

This time, the experimental quantities that should coincide with Bob's marginal clearly depend on Alice's measurement setting $\alpha$. It should be noted that our normalization procedure based on the Fair Sampling Assumption reduces the magnitude

of this behavior, but without removing it completely. If the standard normalization based only on the coincidence counts are used (by computing quantities like $\mathrm{N}^{++}+\mathrm{N}^{-+}$ divided by the total sum of coincides), the visibility of these anomalies are even greater.

\section{Conclusion}

Our result, obtained under the assumption of fair sampling, shows that Bob's marginal probabilities clearly vary with Alice's setting $\alpha$, in violation of the non signalling principle. It logically means that these anomalies imply either the violation of the fair sampling assumption, or the violation of the mo signalling principle, or the violation of both. 

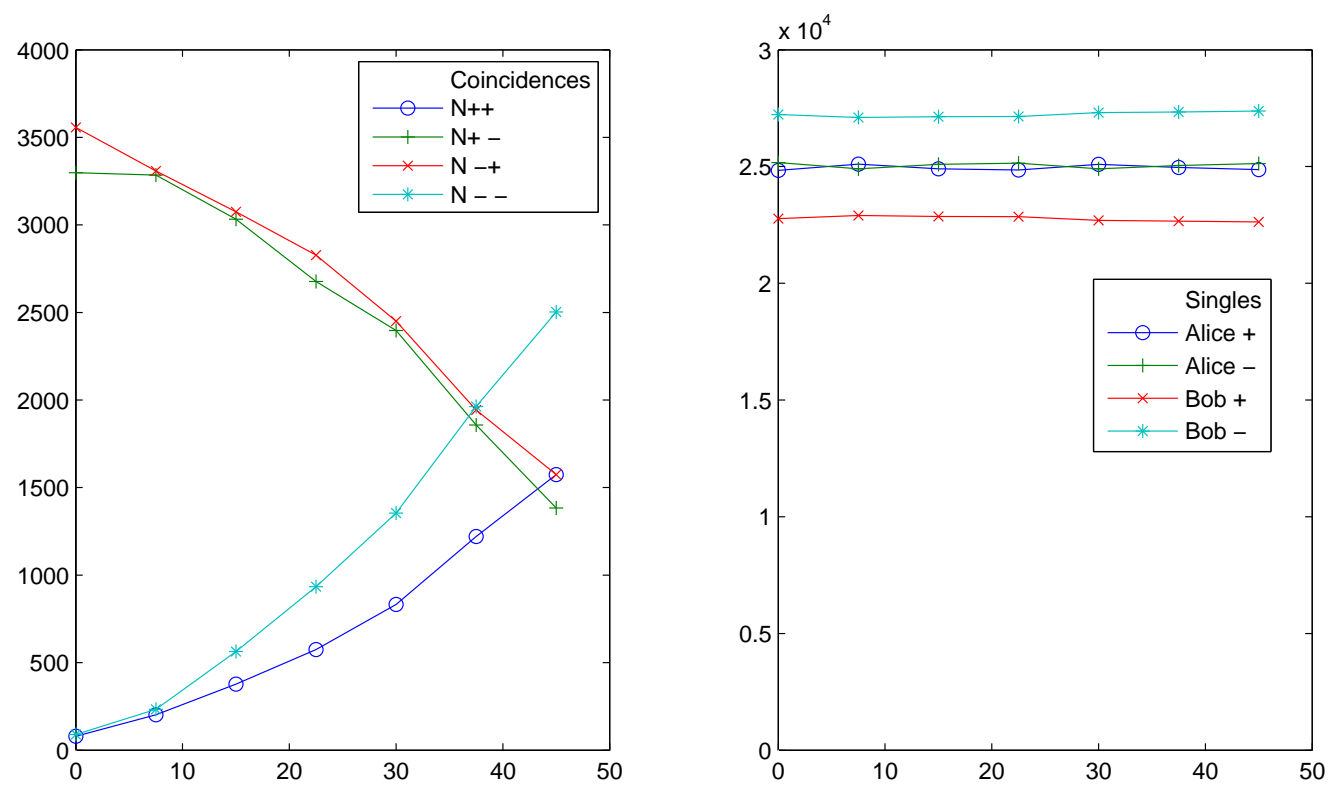

Figure 5. (a) Coincidence counts as Alice varies her measurement angle (in degrees). Note that $N_{++}$and $N_{--}$differ significantly while $N_{+-}$and $N_{-+}$roughly coincide. (Sourcefile: bluesine), (b) Single Counts for Alice and Bob as Alice varies measurement angle $\alpha$. Bob's detector are imbalanced (he gets more clicks in the minus channel).

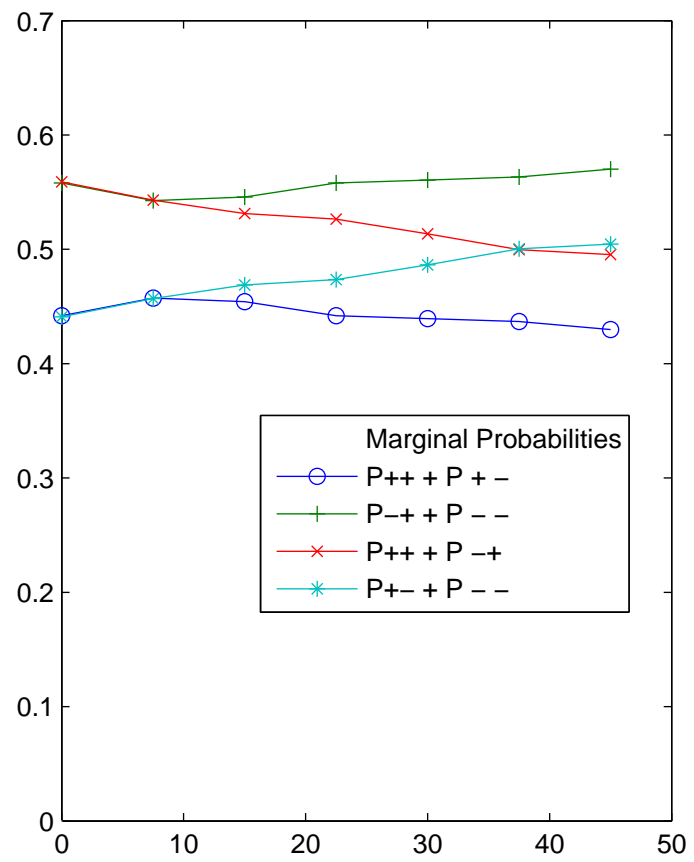

Figure 6. Experimental result that should coincide with the marginal probabilities, assuming Fair Sampling. 
It should be noted that these anomalies cannot be the result of variations or discrepancies in the single count channel efficiencies. The main feature of our normalization procedure is indeed that — once Fair Sampling has been explicitly assumed - the dependence on the channel efficiencies disappear from the final expressions relating Quantum predictions to directly measurable experimental quantities. This is an improvement on the standard normalization procedure, which in general leads to results depending not only the predictions for the considered Quantum state but also on the channel efficiencies.

The no-signalling principle being a fundamental feature of Quantum Mechanics, as well as of Local Realism, the most reasonable interpretation of these anomalies is that the Fair Sampling assumption should be rejected $\boldsymbol{\Phi}$, as it is the only extra assumption that we used to observe them.

In other words, we do not believe that our investigation supports the idea of faster than light signalling, although this possibility cannot be logically excluded. Once the Fair Sampling assumption is rejected, there is no evidence of violation of the nosignalling principle, and therefore no evidence whatsoever of a possible superluminal communication. It should nevertheless be stressed that if one prefers to maintain the Fair Sampling assumption at all cost, the most striking result that can be derived under this assumption is not so much the violation of Bell Inequalities, but rather the violation of no signalling principle.

In any case, our results show that more experiments are definitely required to understand whether this feature is unique or not in EPR experiments.

\section{Acknowledgments}

We are most grateful to Gregor Weihs from Waterloo University (Canada) as he had the courtesy to send us two CDs containing the raw data gathered in Innsbruck between 1997 and 1998 in various version of the EPR setup. This work would not have been possible otherwise. We are also very grateful to Alain Aspect who lend us a precious copy of his PhD. We are grateful to Alain Aspect and Gregor Weihs for stimulating discussions on the structure of statistical data in EPR experiments.

I Note that this can be interpreted in the framework of contextual probabilities $[12,13]$. By choosing a pair of settings $\Pi=(\alpha, \beta)$, we choose choose a selection procedure for pairs of particles. Each selection procedure produces its own ensemble of pairs $S_{\Pi}$, and the statistical properties of $S_{\Pi}$ does depend on $\Pi=(\alpha, \beta)$. It therefore depends explicitly on an experimental context $C_{\Pi}$ that can relate to Bohr's reply to Einstein in 1935. It is not clear how Bohr would have reacted to such an explicit contextuality, but since he would probably have rejected a remote contextuality based on an explicit nonlocality [16], we believe that our interpretation of contextuality based on selection procedure offers a link between Einstein's ensemble interpretation of Quantum Mechanics and Bohr's contextual viewpoint. The point with an explicit contextuality as this one is that Bell's derivation of his famous inequality becomes impossible as it is derived from a fixed probability measure $\rho$. This problem was discussed in [17], p. 95, where generalizations of Bell Inequalities were obtained within the framework of contextuality. Such generalized Bell Inequalities are not violated by experimental data. 
This work was supported by the Profile Mathematical Modelling of Växjö University and the EU-network on QP and Applications.

\section{Predictions of Quantum Mechanics for a non-maximally entangled state}

As an example of the non-signalling principle, we can consider the source of pairs of photons to be represented by a non-maximally entangled state of the form:

$$
|\psi\rangle=\frac{1}{\sqrt{1+p^{2}}}\left[|H\rangle_{1} \otimes|V\rangle_{2}-p|V\rangle_{1} \otimes|H\rangle_{2}\right]
$$

With this state, it is straightforward to obtain the joint probability of detecting a ++ event when polarizers 1 and 2 are oriented at $\alpha$ and $\beta$ respectively. The result is given in quantum mechanics by the Born rule as:

$$
P_{Q T}^{++}(\alpha, \beta)=\mid\left(\left.\left\langle+_{\alpha}\left|\otimes\left\langle+{ }_{\beta}\right|\right) \mid \psi\right\rangle\right|^{2}\right.
$$

The joint probability $P_{Q T}^{\varepsilon_{1}, \varepsilon_{2}}(\alpha, \beta)$ to get the results $\varepsilon_{1}$ and $\varepsilon_{2}$, given the measurement settings $\alpha$ and $\beta$, can be derived explicitly as:

$$
\begin{aligned}
& P_{Q T}^{++}(\alpha, \beta)=\frac{(p \sin \alpha \cos \beta-\cos \alpha \sin \beta)^{2}}{1+p^{2}} \\
& P_{Q T}^{+-}(\alpha, \beta)=\frac{(\cos \alpha \cos \beta+p \sin \alpha \sin \beta)^{2}}{1+p^{2}} \\
& P_{Q T}^{-+}(\alpha, \beta)=\frac{(p \cos \alpha \cos \beta+\sin \alpha \sin \beta)^{2}}{1+p^{2}} \\
& P_{Q T}^{--}(\alpha, \beta)=\frac{(\sin \alpha \cos \beta-p \cos \alpha \sin \beta)^{2}}{1+p^{2}} .
\end{aligned}
$$

It is straightforward to show that the marginal probabilities for this particular source state are:

$$
\begin{aligned}
& P_{Q T}^{++}(\alpha, \beta)+P_{Q T}^{+-}(\alpha, \beta)=\frac{\cos ^{2} \alpha+p^{2} \sin ^{2} \alpha}{1+p^{2}} \\
& P_{Q T}^{-+}(\alpha, \beta)+P_{Q T}^{--}(\alpha, \beta)=\frac{p^{2} \cos ^{2} \alpha+\sin ^{2} \alpha}{1+p^{2}} \\
& P_{Q T}^{++}(\alpha, \beta)+P_{Q T}^{-+}(\alpha, \beta)=\frac{p^{2} \cos ^{2} \beta+\sin ^{2} \beta}{1+p^{2}} \\
& P_{Q T}^{+-}(\alpha, \beta)+P_{Q T}^{--}(\alpha, \beta)=\frac{\cos ^{2} \beta+p^{2} \sin ^{2} \beta}{1+p^{2}},
\end{aligned}
$$

which fulfil the non-signaling principle, as Alice's marginal probabilities depend $\alpha$ alone, while Bob's marginal probabilities depend on $\beta$ alone.

\section{References}

[1] Einstein A, Podolsky B, and Rosen N 1935 Phys. Rev. 47777

[2] Bohm D J 1951 Quantum Theory (Englewood Cliffs: Prentice-Hall)

[3] Bell J S 1964 Physics 1195 
[4] Clauser J F, Horne M A, Shimony A, and Holt R A 1969 Phys. Rev. Lett. 23880

[5] Aspect A, Dalibard J, and Roger G 1982 Phys. Rev. Lett. 49 1804-1807

[6] Weihs G, Jennewein T, Simon C, Weinfurter H, and Zeilinger A 1998 Phys. Rev. Lett. 81 5039-5043

[7] Clauser J F and Shimony A 1978 Rep. Prog. Phys. 41 1881-1927

[8] Adenier G and Khrennikov A Yu 2003 Proc. Conf. Quantum Theory: Reconsideration of Foundations-2 (Växjö Univ. Press)

[9] Tittel W and Weihs G 2001 Photonic Entanglement for Fundamental Tests and Quantum Communication (QIC) 3-56.

[10] Sergienko A V and Jaeger G S 2003 Contemporary Physics 44341

[11] Larsson J -Å 1998 Phys. Rev. A. 5733043308

[12] Khrennikov A Yu 2001 J. Phys. A: Math. Gen. 34 9965-9981

[13] Khrennikov A Yu 2004 Foundations of Physics Letters 17 No 7 p 691

[14] Gisin N 2005 Preprint quant-ph/0512168

[15] Aspect A 1983 PhD. Thesis 2674 (Orsay)

[16] Wiseman H M 2005 Preprint quant-ph/0509061

[17] Khennikov A Yu 2003 Interpretations of Probability (VSP, Utrecht-Boston) 